\title{
Review
}

\section{Treatment strategy for gastric small gastrointestinal stromal tumors}

\author{
Keyu Chen ${ }^{2}$, Yuemin Sun ${ }^{1^{*}}$, Hua Zhang ${ }^{2^{*}}$ \\ ${ }^{1}$ Department of Pancreatic-Gastric Surgery, National Cancer Center, National Clinical Research Center for Cancer, \\ Cancer Hospital, Chinese Academy of Medical Sciences and Peking Union Medical College, Beijing, 100021, China; \\ ${ }^{2}$ Chinese Academy of Medical Sciences and Peking Union Medical College, Beijing, 100730, China
}

*Corresponding authors: Yuemin Sun, NO.17 Panjiayuan Nanli, Chaoyang District, Beijing, 100021, China; E-mail: sunyuemin @ sina.com Tel: 13391860206; Hua Zhang, NO.9 Dongdan Santiao, Dongcheng District, Beijing, 100730, China; E-mail: eyezhanghua@163.com Tel: 13910957617

Received September 28, 2019; Accepted December 17, 2019

With the widespread use of screening endoscopy, more subclinical gastric small gastrointestinal stromal tumors(GS-GISTs) $(<2 \mathrm{~cm}$ in diameter) are detected. Most of them exhibit benign clinical behavior and can be followed by periodical endoscopic ultrasonography. But some of them have malignant potential and needed surgery resection. A preoperative assessment of the malignant potential is difficult in many cases. With the improvement of laparoscopic techniques, some GS-GISTs are recommended for resection. Endoscopic resection is not routinely indicated, because of the risk for tumor cell spillage and seeding.

Key Words: gastric small gastrointestinal stromal tumors(GS-GISTs); endoscopic ultrasonography (EUS); laparoscopic surgery

\section{INTRODUCTION}

Gastrointestinal stromal tumors (GISTs) are considered potentially malignant neoplasms arising from mesenchymal cells. It is estimated that the annual incidence of GISTs is 10 per million population. Most of them occur from the stomach $(60 \%)$, followed by the small intestine $(30 \%)$, and the colon/rectum (5\%). ${ }^{1}$ The proliferation of GISTs is primarily caused by activating mutations in KIT or PDGFRA, both of which encode receptor tyrosine kinases. ${ }^{2}$ GISTs are often diagnosed postoperatively. However, it may be diagnosed preoperatively, usually by endoscopic ultrasound (EUS)-guided fine-needle aspiration (EUS-FNA). With the widespread use of screening endoscopy, more small gastrointestinal stromal tumors have been reported especially in the stomach. The gastric submucosal tumors measuring $<2 \mathrm{~cm}$ in diameter are identified in $0.15 \%$ of middle-aged adults and nearly one-half are GISTs. ${ }^{3}$ The natural history of these GISTs and the optimal treatment strategy are unclear.

\section{TREATMENT STRATEGY FORGS-GISTS}

In the most report, Small-GISTs are defined as those measuring $<2$ $\mathrm{cm}$ in diameter which often be discovered incidentally during screening endoscopy. Rossi has suggested the activity of GS-GISTs is quite low, especially when they measure $<1 \mathrm{~cm}^{4}{ }^{4}$ Therefore guidelines come from NCCN, ESMO and Asian all suggest that GS-GISTs with high-risk features(irregular borders, cystic spaces, ulceration, echogenic foci, internal heterogeneity on EUS, and growing during follow-up) should be resected. Whereas GS-GISTs without those characters can be followed by EUS. ${ }^{2,5,6}$

\section{THERAPEUTIC APPROACH}

For small gastric GISTs, open or laparoscopic surgery is standard therapy. The surgical methods are selected according to tumor size, location, growth pattern, and an oncology team. The principle of surgery includes complete resection that avoids tumor rupture. Lymphadenectomy is not necessary for most patients. If there are enlarged lymph nodes, limited dissection is indicated. Modest surgery, such as wedge resection, should be considered to preserve organ functions when tumors are resectable with negative surgical margins. ${ }^{7}$ After resection, pathologic examination for diagnosis and risk assessment is mandatory, and gene detection is also recommended for high-risk GISTs.

\section{Open Surgery}

The standard operation for GIST is complete resection with sufficient surgical margins by laparotomy. However,with the

This is an open access journal, and articles are distributed under the terms of the Creative Commons Attribution-NonCommercial-ShareAlike 4.0 License, which allows others to remix, tweak, and build upon the work non-commercially, as long as appropriate credit is given and the new creations are licensed under the identical terms.

For reprints contact: weda-h@ weda-h.org

How to cite this article: Chen KY, Sun YM, Zhang H. Treatment strategy for gastric small gastrointestinal stromal tumors. $J A D V$ HEALTH 2019; 1(4): 279-280. 
development of minimally invasive techniques, laparotomy has been used less in the treatment of GS-GISTs.

\section{Laparoscopic Surgery}

Several retrospective studies have suggested that laparoscopic surgery for gastric GISTs $<5 \mathrm{~cm}$ may be feasible and safe, with less invasiveness, better short-term results, and long-term oncologic outcomes similar to those achieved with open surgery. ${ }^{8}$ The most common laparoscopic surgery for gastric-GISTs currently includes laparoscopic wedge resection, laparoscopic transgastric surgery, and laparoscopic intragastric surgery.

Laparoscopic surgery usually is used to lesions on the greater curvature, anterior wall of the stomach. When tumors are located on the lesser curvature, gastroesophageal junction and pylorus, the resection may result in the deformity of the gastral cavity. Yong $\mathrm{Li}^{9}$ have reported the total laparoscopic intragastric technique for cardiac endophytic gastric-GISTs.Unlike the endoscopic resection, the incision inside the stomach can be continuously sutured with a 3-0, no-tied line. Also full-layer suturing can be fulfilled if necessary.

\section{Endoscopic resection}

When the lesions are located in the muscularis mucosae, endoscopic submucosal dissection(ESD) may be a safe, cost-effective, and minimally invasive option. However, when the lesions reach the muscularis propria, a full-thickness resection is needed. The reported rates of negative (R0) resection range from $75 \%$ to $100 \%$ by endoscopic submucosal dissection. ${ }^{10}$

Although there are many endoscopic resection methods(such as endoscopic full-thickness resection, muscularis excavation and submucosal tunneling resection) for GS-GISTs. ${ }^{11}$ But in guidelines, these techniques are not be recommended because of the risk of tumor cell spillage and seeding and still require evidence of oncologic outcomes.

\section{Laparoscopic and Endoscopic Cooperative Surgery (LECS)}

Besides the location factors, the laparoscopic approach is sometimes difficult in resecting GS-GISTs with intraluminal growth, because the identification of lesions from the outside of the stomach is uncertain. Conversely, localization of these lesions is straightforward under endoscopic observation.Therefore, endoscopic full-thickness resection with laparoscopic suturing may be an ideal method for accurate resection. This technique is reported to be feasibility, safety, and short-term efficacy, especially for small gastric GISTs near the gastroesophageal junction and pylorus to preserve junctional functions, ${ }^{12}$ although long-term outcomes are awaited.

\section{CONCLUSION}

GISTs are considered to be potentially malignant tumors. There are large numbers of subclinical GS-GISTs being discovered in screening endoscopy. Most of them exhibit nice clinical behavior and the only exceptional case may progress. GS-GISTs cannot be identified as benign or malignant by radiographic and endoscopic imaging. Although EUS-FNA is recommended for these tumors, it is difficult to implement in clinical practice. According to the current guidelines come from NCCN, ESMO and Asian, GS-GISTs with high-risk features(irregular borders, cystic spaces, ulceration, echogenic foci, internal heterogeneity on EUS, and growing during follow-up) should be resected. Whereas GS-GISTs without those characters can be followed by EUS. But some Chinese reports suggest that they can develop disease progression and metastasis. ${ }^{13-15}$ Moreover, the annual endoscopic review also brings ordeal and financial burdens. So we recommend that surgery should be performed if GS-GISTs measure $>1 \mathrm{~cm}$, especially when they located in the cardia. With the development of surgical instruments, the trauma is becoming smaller. If the technique is enough safer, we can remove them just like removing the colonic polyps. Cost-effectiveness also should be considered in the treatment of GS-GISTs.

\section{Funding Support}

This work was supported by the National Natural Science Foundation of China (No. 51890894).

\section{REFERENCES}

1. Joensuu H, Hohenberger P, Corless CL. Gastrointestinal stromal tumour. Lancet2013; 382(9896): 973-83.

2. von MM, Randall RL, Benjamin RS, et al. Soft Tissue Sarcoma, Version 2.2018, NCCN Clinical Practice Guidelines in Oncology. J Natl Compr Canc Netw 2018; 16(5): 536-563.

3. Hedenbro JL, Ekelund M, Wetterberg P. Endoscopic diagnosis of submucosal gastric lesions. The results after routine endoscopy. Surg Endosc 1991; 5(1): 20-3.

4. Rossi S, Gasparotto D, Toffolatti L, et al. Molecular and clinicopathologic characterization of gastrointestinal stromal tumors (GISTs) of small size. Am J Surg Pathol 2010; 34(10): 1480-91.

5. Koo DH, Ryu MH, Kim KM, et al. Asian Consensus Guidelines for the Diagnosis and Management of Gastrointestinal Stromal Tumor. Cancer Res Treat 2016; 48(4): 1155-1166.

6. Casali PG, Abecassis N, Aro HT, et al. Gastrointestinal stromal tumours: ESMO-EURACAN Clinical Practice Guidelines for diagnosis, treatment and follow-up. Ann Oncol 2018; 29(Suppl4): iv267.

7. Nishida T, Blay JY, Hirota S, Kitagawa Y, Kang YK. The standard diagnosis, treatment, and follow-up of gastrointestinal stromal tumors based on guidelines. Gastric Cancer 2016; 19(1): 3-14.

8. Bischof DA, Pawlik TM. Open Versus Minimally Invasive Resection of Gastric GIST: A Multi-Institutional Analysis of Short- and LongTerm Outcomes: A Reply. Ann Surg Oncol 2017; 24(Suppl 3): 626.

9. Lyu Z, Yang Z, Wang J, Hu W, Li Y. Totally Laparoscopic Transluminal Resection for Gastrointestinal Stromal Tumors Located at the Cardiac Region. Ann Surg Oncol 2018; 25(8): 2218-2219.

10. Ito S, Tsuchitani Y, Kim Y, et al. A gastrointestinal stromal tumor of the jejunum presenting with an intratumoral abscess: A case report and a literature review. Int J Surg Case Rep 2018; 48: 65-68.

11. Zhang Y, Ye LP, Zhu LH, Zhou XB, Mao XL, Ding JX. Endoscopic muscularis excavation for subepithelial tumors of the esophagogastric junction originating from the muscularis propria layer. Dig Dis Sci 2013; 58(5): 1335-40.

12. Hiki N, Yamamoto Y, Fukunaga T, et al. Laparoscopic and endoscopic cooperative surgery for gastrointestinal stromal tumor dissection. Surg Endosc 2008; 22(7): 1729-35.

13. Huang Z, Li Y, Zhao H, Zhao JJ, Cai JQ. Prognostic factors and clinicopathological characteristics of small gastrointestinal stromal tumor of the stomach: A retrospective study of 31 cases.Chin J Chin Oncol2013; 40(12): 730-732.

14. Ma JJ, Zhang YY, Bai L.Clinicopathological characteristics and management strategy of small gastrointestinal stromal tumors.Chin J Dig Endose2016; 33(12): 834-837.

15. Wu LY, Peng CY, et al. Evaluation of clinical treatment of primary small gastric gastrointestinal stromal tumors: a retrospective sing-center study.2016; 33(7): 442-446. 